\title{
Kawasaki Disease; A Review Article
}

\author{
Reza Shiari ${ }^{1, *}$ \\ ${ }^{1}$ Department of Pediatric Rheumatology, Mofid Children's Hospital, Shahid Beheshti University of Medical Sciences, Tehran, IR Iran \\ ${ }^{*}$ Corresponding author: Reza Shiari, Department of Pediatric Rheumatology, Mofid Children's Hospital, Shahid Beheshti University of Medical Sciences, Shariati Ave, Hosseinieh \\ Ershad, Tehran, IR Iran. Tel/Fax: +98-2122227033, E-mail: shiareza@yahoo.com.
}

Received: April 26, 2013; Revised: Jun 19, 2013; Accepted: July 03, 2013

Kawasaki disease is believed to be the commonest vasculitis of medium vessels in Asian children. However, the majority of children with Kawasaki disease continue to remain undiagnosed perhaps because of the lack of awareness amongst pediatricians. The clinical features of Kawasaki disease can be confused with other common conditions such as Scarlet fever, Stevens Johnson syndrome, Measles fever, systemic onset JIA, etc. Development of coronary artery abnormalities is the hallmark of Kawasaki disease and accounts for most morbidity and mortality associated with the disease. Quick recognition of the disease and early treatment with intravenous immunoglobulin results in a significant decrease in the occurrence of coronary artery abnormalities.

This review emphasizes that Kawasaki disease should be considered in differential diagnosis of all febrile illnesses in young children.

Keywords: Mucocutaneous Lymph Node Syndrome; Vasculitis; Aneurysm; Kawasaki Disease; Coronary Vessels

\section{Introduction and History}

Kawasaki disease (KD) or acute mucocutaneous lymph node syndrome is a systemic vasculitis with unknown origin and one of the causes of acquired heart disease in Asian children (1). KD was first reported in 1967 by professor Tomisaku Kawasaki (2). He described the clinical signs and symptoms of 50 Japanese children with acute mucocutaneous lymph node syndrome (2). One year later, Yamamoto T, published the electrocardiogram abnormalities of KD patients (3). Finally, Japanese physicians established the relationship between KD and coronary vasculitis in 1972 (4). Since that time, KD has been described in many countries and mostly in Asia (5).

\section{Etiology}

The cause of Kawasaki disease still remains unknown. It is supposed that there is a stimulation of the immune system after an infection in a genetically predisposed person (6). Although, no bacteria or virus has been consistently found yet, an infectious cause is highly suspected (7). Some of the infectious agents proposed are Parvovirus (8), Staphylococcus aureus (9), Epstein-Barr virus (10), Chlamydia (11), and Mycobacteria (12). The occurrence of this condition in young children further corroborates this hypothesis. Genetics may illuminate why the risk of KD in a child with a history of affected siblings or parents, is higher than in the all-purpose population $(13,14)$. There is still significant disagreement about the mechanism of immune system that triggers in patients with KD.

\section{Clinical Manifestations of KD}

The diagnosis of KD is based on the clinical picture of the disease. At the moment, there is no specific test for diagnosis of KD. However, the diagnostic clinical criteria may help clinicians for diagnosis of $\mathrm{KD}$ (7) (Table 1). It is important to know that the diagnostic criteria are defined once other diseases with similar findings have been ruled-out $(7,15)$. Kawasaki disease has three stages: acute febrile, subacute, and convalescent. The acute febrile phase is characterized by fever. The fever in KD is hectic and is usually higher than $39^{\circ} \mathrm{C}\left(102{ }^{\circ} \mathrm{F}\right)$ and is unresponsive to antipyretics and antibiotic therapy (15). If a child with KD is untreated, his/her fever and illness typically lasts for 1 to 2 weeks. Conjunctivitis (non purulent and bulbar) (Figure 1), oropharyngeal changes (erythema, cracking, or fissuring of the lips, strawberry tongue, and non-exudative erythema of the oropharynx), and polymorphous rash (except bullous or vesicular eruption) are the most common clinical features of $\mathrm{KD}$ in its acute phase $(7,15)$. The perineal desquamation and peripheral extremity changes (erythema or edema of the palms or soles) occurs during the acute phase of $\mathrm{KD}$ (16). Cervical lymphadenopathy is not common in $\mathrm{KD}$. It is usually found as a unilateral, firm and non-fluctuant node with a diameter greater than $1.5 \mathrm{~cm}$, during acute phase

Implication for health policy/practice/research/medical education:

Kawasaki disease is believed to be the commonest vasculitis of medium vessels in Asian children. However, the majority of children with Kawasaki disease continue to remain undiagnosed perhaps because of the lack of awareness amongst pediatricians. This review emphasized that Kawasaki disease should be considered in the differential diagnosis of all febrile illnesses in young children.

Copyright @ 2014, Pediartric Infections Research Center. This is an Open Access article distributed under the terms of the Creative Commons Attribution License (http://creativecommons.org/licenses/by/3.0), which permits unrestricted use, distribution, and reproduction in any medium, provided the original work is properly cited. 
of the disease. The cervical adenopathy needs to be considered in children who are not responding to antibiotic therapy (17).

When fever and other signs of acute phase start to vanish the subacute phase initiates. During this phase, the child is at the highest risk for sudden death because of coronary vasculitis in combination with significant thrombocytosis and hypercoagulable state. Around weeks 4 to 6 when all signs of illness have disappeared, the convalescent phase begins, and it remains until the acute phase reactants become normal. Deep transverse grooves across the nails (Beau's lines) within 1 to 2 months after the onset of fever have been described (18) (Figure 2). Erythema and induration at the previous site of BCG vaccine are commonly seen (19).

\begin{tabular}{l} 
Table 1. Classification Criteria for Kawasaki Disease \\
\hline Classic/Complete KD ${ }^{\mathrm{a}}$ \\
Fever persisting for 5 days and the presence of 4 from 5 of the \\
following clinical criteria: \\
Conjunctivitis (Bilateral, non-exudative, bulbar) \\
Oropharyngeal changes with any of the following: \\
Strawberry tongue \\
Diffuse erythema of the oropharyngeal mucosa \\
Erythema or cracking of the lips \\
Cervical lymphadenopathy(>1.5 cm diameter, usuallyunilateral) \\
Rash (Polymorphous) \\
Peripheral extremity changes with any of the following: \\
Erythema or edema of the palms or soles \\
Periungal desquamation in the subacute phase \\
Incomplete KD \\
Fever_5 days with 2 or 3 of the above clinical criteria \\
Atypical KD \\
Patients who fulfill the criteria for KD with a clinical fea- \\
ture that is not usually seen with KD (e.g., a patient with KD \\
with pulmonary or renal involvement.) \\
\hline a Abbreviation: KD; kawasaki disease
\end{tabular}

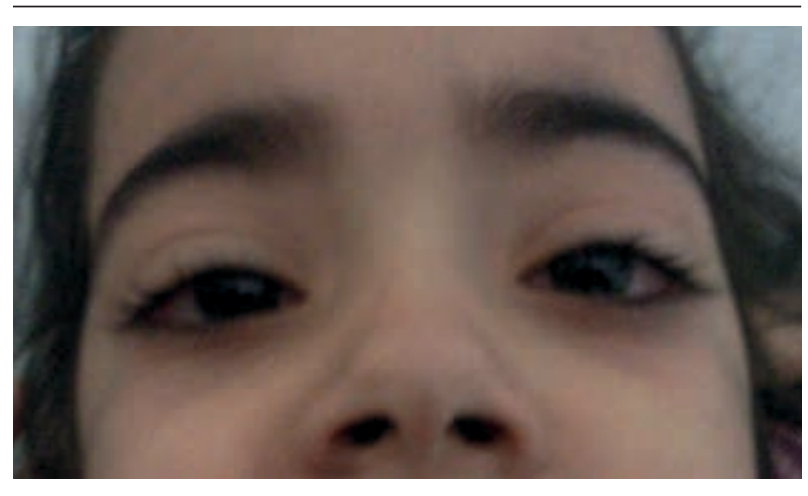

Figure 1. Bilateral, Non purulent Conjunctivitis
Arthralgia, arthritis (oligo or polyarticular), abdominal pain, vomiting, diarrhea, hepatitis, hydrops of the gallbladder, irritability, urethritis, aseptic meningitis, facial palsy, sterile pyuria and otitis media are some of the less common clinical findings in children with $\operatorname{KD}(20,21)$.

Cardiac abnormalities in the acute phase are as following: tachycardia out of quantity to the fever, decreased $\mathrm{R}$-wave voltage, changes in the ST and T waves, and pro

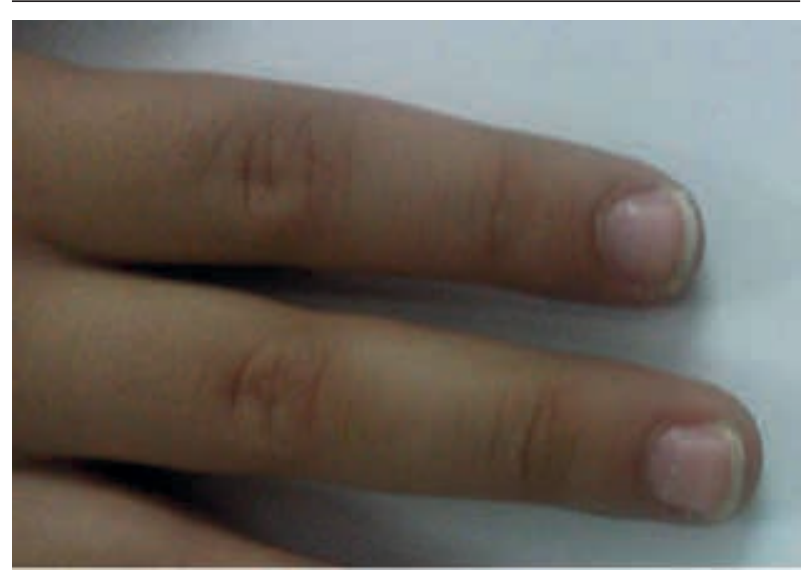

Figure 2. Beau's Line

longed PR or QT intervals in electrocardiography, pericardial effusion, myocarditis, congestive heart failure, and mitral regurgitation $(22,23)$.

Laboratory findings may include neutrophilia, anemia, thrombocytosis, elevated erythrocyte sedimentation rate, elevated serum transaminase levels, hypoalbuminemia, positive C-reactive protein test result and an elevated serum $\alpha 1$-antitrypsin level.

\section{Laboratory Findings}

Although, laboratory examinations in the acute phase of KD are nonspecific, however, in children who do not fulfill diagnostic criteria, these may support the diagnosis. Acute phase reactants [erythrocyte sedimentation rate (ESR) and C-reactive protein (CRP)] are almost always increased. Increased leukocytosis (neutrophil dominant) is frequent $(24,25)$. Thrombocytosis as a characteristic feature of KD usually occurs during the 2 weeks of the illness. Increased serum transaminase levels, hypoalbuminemia and sterile pyuria are common $(24,25)$.

Thrombocytopenia is uncommon in $\mathrm{KD}$, but it is considered as a risk factor for the development of aneurism. Also, leukopenia and thrombocytopenia can be seen in KD with hemophagocytic lymphohistiocytosis syndrome (macrophage activation syndrome) $(26,27)$.

$\mathrm{N}$-terminal pro-B-type natriuretic peptide (NT-proBNP) is a cardiovascular biomarker. It is associated with cardiomyocyte stress, markers of inflammation, oxidative stress, and echocardiographic measurements. Presence of NT-proBNP suggests diastolic dysfunction (28). Some 
researchers propose that NT-proBNP may be an excellent biomarker for supporting the diagnosis of $\mathrm{KD}(29)$. These investigators showed that NT-proBNP was increased in both patients with KD who fulfilled the criteria and those who did not, compared with febrile controls (29). They suggested that NT-proBNP might be a valid adjunctive diagnostic test for patients with KD particularly those with incomplete diagnostic criteria.

\section{Management}

It is necessary for all patients who are suspected of having KD, to admit at hospital for more evaluation and appropriate treatment. Two-dimensional echocardiogram and ECG have to be performed for all admitted patients. The most common findings in echocardiography are as followings: myocarditis, pericarditis, valvulitis, valvular regurgitation, coronary artery abnormalities, aneurysms of medium-size noncoronary arteries, conduction system involvement, and Raynaud's phenomenon (23, 30, 31). Coronary artery aneurysm is the main complication of KD, which develops in up to $25 \%$ of untreated children. This risk decreases with appropriate treatment to less than $5 \%(30,31)$. Myocardial infarction, ischemic heart disease and sudden death are the main problems of coronary artery abnormalities (CAA). Hence, treatment of KD patients after the acute phase of the disease is necessary.

Although coronary artery abnormalities (CAA) develop more commonly in the subacute phase, it has also been reported in the acute phase as early as 3 days from onset of disease (20).

Patients with uncomplicated KD should go through an echocardiogram at time of diagnosis, at 2 weeks, and 8 weeks after disease onset $(7,30)$. For patients with previously normal studies, repeated echocardiography should be performed optionally beyond 8 weeks. However, more frequent echocardiograms are required in those with CAA $(7,32)$. Other arteries including the axillary, renal, and iliac arteries may also be involved in $\mathrm{KD}$ (33).

The clinical and laboratory findings that are associated with an increased risk of CAA are as follows: prolonged fever, persistent fever after treatment, male gender, extremes of age, delay in diagnosis, thrombocytopenia, leukocytosis, bandemia, anemia, very increased or persistently increased in ESR or CRP, and hypoalbominemia $(7,25,34)$.

\section{Incomplete and Atypical KD}

Patients who have a fever for at least five days but fewer than 4 clinical criteria are diagnosed as having incomplete KD (7). It is more common in infants, and those older than 9 years of age. Incomplete KD should not be judged as mild KD because the risk of CAA in these children is similar with classic KD (35). The expression of atypical Kawasaki disease should be reserved for patients who have a problem, such as renal impairment or uveitis that are generally not seen in Kawasaki disease.

Infants younger than 6 months of age are more likely to have an incomplete presentation of KD because of poor laboratory profile and delay in diagnosis. Their response to treatment with intravenous immunoglobulin (IVIG) is late. Therefore, risk of CAA is higher in these infants (36). An algorithm has been proposed by the American heart association (AHA) for incomplete $\mathrm{KD}$ in 2004. This algorithm uses the data of the clinical, laboratory, and echocardiographic features for diagnosis of KD in children with the incomplete disease. According to the AHA algorithm, a 6 month old infant or younger, who is on the seventh day of fever or greater, without an origin, should have laboratory tests looking for signs of inflammation. If systemic inflammation is discovered, with an increased CRP of $3 \mathrm{mg} / \mathrm{dL}$ or greater or ESR of $40 \mathrm{~mm} / \mathrm{h}$ or greater, then an echocardiogram should be considered even if no clinical criteria are present. If abnormal findings are seen on echocardiogram, treatment of KD is suggested. In children older than 6 months of age with incomplete criteria, if their clinical characteristics are consistent with KD, an ESR and CRP are also advised. If no signs of inflammation are found, patients need to be reevaluated within 48 hours and the algorithm will be repeated. If there is evidence for inflammation, supplemental laboratory testing (low albumin level, anemia, increased alanine aminotransferase level, increased platelet count after the seventh day, increased white blood cell count, and pyuria) is suggested. If the patient fulfills at least 3 supplemental laboratory criteria, AHA suggests treatment for KD. If the patient has fewer than 3 supplemental laboratory criteria, an echocardiogram is advised. Then, if the echocardiogram is abnormal, treatment is suggested. However, if the echocardiogram was negative and fever persists, AHA suggests repeating an echocardiogram and consulting a KD expert (7).

Clinical characteristics that would help exclude the diagnosis include, exudative conjunctivitis or pharyngitis, discrete intra-oral lesions, bullous or vesicular rash, and generalized lymphadenopathy.

\section{Treatment}

Treatment of $\mathrm{KD}$ in the acute phase focuses on reduction of coronary artery wall inflammation, inhibition of platelet activation, and prevention of arterial thrombosis. Chronic or long-term management is dependent on coronary artery changes and focuses on prevention of myocardial ischemia and infarction. The AHA recommends that all children suspected to have KD need to be treated with a single dose of 2 $\mathrm{g} / \mathrm{kg}$ of intravenous immunoglobulin (IVIG) infused over 12 hours plus Aspirin at a dose of 80 to $100 \mathrm{mg} / \mathrm{kg}$ per day (anti inflammatory dose) in 4 divided doses. Treatment has to start within the first 10 days of illness. However, IVIG should also be given to patients who present after the 10th day of illness 
if fever is still continued, or aneurysms are observed with evidence of persistent inflammation. Once the child is afebrile for 48 to 72 hours, Aspirin may be switched to a low-dose (or antiplatelet dose), 3-5 mg/kg/day and will discontinue after 6 to 8 weeks when there is a normal echocardiogram. Nevertheless, the low-dose of Aspirin will continue if the echocardiogram shows CAA (7) (Table 2). Hemoglobin should be watchfully monitored after IVIG retreatment (37). Measles and varicella vaccinations should be deferred for 11 months after the patient has received a high-dose IVIG for $\mathrm{KD}$ (7).

High-dose of Aspirin has been used for all the large IVIG trials conducted in the United States. However, the highdose of Aspirin is not used in Japan. Although high-dose of Aspirin has been shown to shorten the duration of fever compared with moderate-dose or low-dose of Aspirin, it is ambiguous if it has any effect on CAA (38).

Table 2. Treatment of Kawasaki Disease

\section{Standard Treatment}

IVIG $2 \mathrm{~g} / \mathrm{kg}$ as a single infusion over $12 \mathrm{~h}$ plus Aspirin 80-100 $\mathrm{mg} / \mathrm{kg}$ per day (oral) in 4 divided doses (keep until afebrile for at least 48-72 h)

Once afebrile for $48-72 \mathrm{~h}$, decrease doses of Aspirin to $3-5 \mathrm{mg} / \mathrm{kg} / \mathrm{d}$ (maintain until $6 \mathrm{wk}$ to $8 \mathrm{wk}$, Echo is normal; if abnormal, continue Aspirin)

\section{Treatment of IVIG Resistance}

Re-treat with second dose of IVIG if fever persists beyond $48 \mathrm{~h}$ from completion of IVIG

Give IVIG $2 \mathrm{~g} / \mathrm{kg}$ as a single infusion over $12 \mathrm{~h}$

\section{If no response to second IVIG}

Methylprednisolone intravenously pulse $30 \mathrm{mg} / \mathrm{kg}$ over $3 \mathrm{~h}$, once daily for 1-3 days

\section{If no response to the above}

If Methylprednisolone was given, then try Infliximab $5 \mathrm{mg} / \mathrm{kg}$ intravenously

Another option: give oral prednisone 1-2 mg/kg/day; continue until CRP has normalized; once normal taper over 2 wk

Other options: Cyclosporin A, Cethotrexate, Cyclophosphamide

\section{Treatment of Resistant KD}

There is no advantage for treating patients with IVIG on day 4 or earlier, because this may result in IVIG retreatment (39). KD patients persist with fever or redevelop fever beyond 48 hours after completion of IVIG; the recommendation is to administer a second dose of $2 \mathrm{~g} / \mathrm{kg}$ of IVIG (7).

It is well known that prolonged fever and refractory disease are risk factors for the development of CAA. Regardless of retreatment with IVIG, there are still approximately $3 \%$ to
$4 \%$ who do not respond (40). For these patients, corticosteroids may be considered. Because $\mathrm{KD}$ is a systemic vasculitis and corticosteroids are the treatment of choice for most vasculitides, it is sensible to consider corticosteroids for KD. A meta-analysis of corticosteroid therapy plus Aspirin showed encouraging results for its use (41). Generally corticosteroids don't have any negative effect on KD and therefore it should be considered for treatment of children.

Table 3. Key Points in KD

Diagnosis of $\mathrm{KD}^{\mathrm{a}}$ is based on a set of clinical features.

Children with KD are often more irritable than children with other febrile illnesses.

Gastrointestinal complaints, including diarrhea, vomiting, and abdominal pain, occur in approximately one third of patients.

Arthritis or arthralgia developing after the 10th day of illness favors large weight-bearing joints.

Approximately $50 \%$ of patients have $\mathrm{WBC}>15000 / \mathrm{mm}^{3}$.

Mild to moderate elevations in serum transaminases occur in $\leq 40 \%$ of patients.

A moderately to markedly elevated CRP or ESR, which is almost universally seen in children with KD, is uncommon in viral infections.

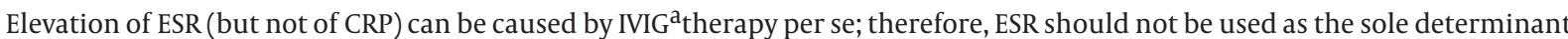
of the degree of inflammatory activity in IVIG-treated patients.

Hypoalbuminemia is common and is associated with more severe and more prolonged acute disease.

Exudative conjunctivitis or pharyngitis, discrete intra-oral lesions, bullous or vesicular rash, and generalized lymphadenopathy are the clinical characteristics that would help exclude the diagnosis of KD.

Infants of 6 to 12 months are more prone to developing 'incomplete' Kawasaki disease and coronary artery aneurysms.

${ }^{a}$ Abbreviations: KD; kawasaki disease, IVIG; intravenous immunoglobulin 


\section{Another Treatment Decision}

Plasmapheresis has been used during the acute phase of KD. It should be performed at an early stage of disease (42), but the risks of bleeding and exposure to blood products is high. Therefore it is not recommend any more. A platelet glycoprotein IIb/IIIa receptor inhibitor (Abciximab), was shown to be effective in patients with large aneurysms during the acute or subacute phases of KD (43). Monoclonal antibodies directed against tumor necrosis factor- $\alpha$, for example infliximab, have been considered in children who are resistant to IVIG therapy, however, their efficacy in decreasing the risk of coronary artery aneurysms is unproved $(44,45)$.

Cytotoxic agents such as Cyclosporin A, Methotrexate, and Cyclophosphamide, in combination with steroids, have been suggested for patients with refractory disease (46-48). However, for most patients, the risk of therapy needs to be evaluated before using these medications.

\section{Long-term Management and Follow-up of KD}

Periodic checkup and counseling for cardiovascular risk factors is recommended for all children who have Kawasaki disease. For children without coronary artery changes, 6 to 8 weeks follow up seems to be enough. However, children with CAA will require life-long pharmacological therapy and follow up (7).

Five risk levels have been created by a consensus of experts and stratified for long-term management of children with Kawasaki disease (7). In risk level one there are no coronary artery changes. In risk level two, transient coronary artery ectasia or dilation occurs, however, these disappear within the first 6 to 8 weeks after the onset of the disease. In both risk levels one and two, no pharmacological therapy or diagnostic or invasive testing is required beyond the initial 6 to 8 weeks after the onset of the disease. An isolated small to medium coronary artery aneurysm is found in at least one coronary artery in risk level three. Therefore, long-term antiplatelet therapy with low-dose of Aspirin (3 to $5 \mathrm{mg} / \mathrm{kg} /$ day) should be recommended until the aneurysm regresses. An annual electrocardiogram (ECG) and echocardiograph is recommended for all children, along with a biennial stress test with myocardial perfusion imaging for children older than 10 years. Angiography should be performed if the noninvasive test suggests ischemia. In risk level four, at least one large coronary aneurysm is present, including giant aneurysms or any size coronary artery aneurysm that contains multiple or complex aneurysms without obstruction. In this level, long-term antiplatelet therapy plus low-dose Aspirin is required. For children with giant aneurysms, adjunctive Warfarin therapy with a target INR of 2 to 2.5 is recommended. Low molecular weight Heparin may be used as an alternative to Warfarin. Fi- nally, children at risk level V have coronary artery obstruction. Long-term antiplatelet therapy with low-dose Aspirin with or without adjunctive Warfarin therapy is necessary for patients who have coronary artery obstruction. Using Beta-blockers (Propranolol 1 to $2 \mathrm{mg} / \mathrm{kg} /$ day) may be considered for these patients to reduce myocardial oxygen consumption. In both risk levels four and five, a biannual ECG and echocardiography, an annual stress test, are recommended for all patients. In addition, a cardiac catheterization with coronary angiography should be performed within 6 to 12 months after recovery from acute disease and repeated again if noninvasive tests or clinical or laboratory findings are suggestive of ischemia.

\section{Conclusion}

Management of children with KD especially in incomplete cases is still a challenging issue. It is important to use experience besides knowledge together to perform diagnosis. Recent biomarkers and genetic studies seem promising to help clinicians for earlier diagnosis, and treatment.

\section{Acknowledgements}

The author wishes to express his gratitude to Prof. Ab. Karimi for his precious assistance, support and guidance.

\section{Authors' Contribution}

First Author Dr. Reza Shiari is responsible for all parts of manuscript.

\section{Financial Disclosure}

There is no financial disclosure.

\section{Funding/Support}

There is no support for this study.

\section{References}

1. Yanagawa H, Nakamura Y, Ojima T, Yashiro M, Tanihara S, Oki I. Changes in epidemic patterns of Kawasaki disease in Japan. Pediatr Infect Dis J.1999;18(1):64-6.

2. Kawasaki T. [Acute febrile mucocutaneous syndrome with lymphoid involvement with specific desquamation of the fingers and toes in children]. Arerugi. 1967;16(3):178-222.

3. Yamamoto T, Kimura J. [Acute febrile mucocutaneous lymph node syndrome (Kawasaki): subtype of mucocutaneous ocular syndrome of erythema multiforme complicated with carditis]. .Jpn J Pediatr. 1968;21:336-9.

4. Shigematsu I. [Epidemiology of mucocutaneous lymph node syndrome].JJpn Pediatr Soc. 1972;76:695-6.

5. Nakamura Y, Yanagawa H. The worldwide epidemiology of Kawasaki disease. Prog Pediatr Cardiol. 2004;19:99-108.

6. Yeung RS. Kawasaki disease: update on pathogenesis. Curr Opin Rheumatol. 2010;22(5):551-60.

7. Newburger JW, Takahashi M, Gerber MA, Gewitz MH, Tani LY, Burns JC, et al. Diagnosis, treatment, and long-term management of Kawasaki disease: a statement for health professionals from the Committee on Rheumatic Fever, Endocarditis and Kawasaki Dis- 
ease, Council on Cardiovascular Disease in the Young, American Heart Association. Circulation. 2004;110(17):2747-71.

8. Nigro G, Zerbini M, Krzysztofiak A, Gentilomi G, Porcaro MA Mango T, et al. Active or recent parvovirus B19 infection in children with Kawasaki disease. Lancet. 1994;343(8908):1260-1.

9. Leung DY, Meissner HC, Fulton DR, Murray DL, Kotzin BL, Schlievert PM. Toxic shocksyndrometoxin-secreting Staphylococcus aureus in Kawasaki syndrome. Lancet. 1993;342(8884):1385-8.

10. Kikuta H, Sakiyama Y, Matsumoto S, Hamada I, Yazaki M, Iwaki T, et al. Detection of Epstein-Barr virus DNA in cardiac and aortic tissues from chronic, active Epstein-Barr virus infection associated with Kawasaki disease-like coronary artery aneurysms. $J$ Pediatr. 1993;123(1):90-2.

11. Normann E, Naas J, Gnarpe J, Backman H, Gnarpe H. Demonstration of Chlamydia pneumoniae in cardiovascular tissues from children with Kawasaki disease. Pediatr Infect Dis J. 1999;18(1):72-3.

12. Hsu YH, Wang YH, Hsu WY, Lee YP. Kawasaki disease characterized by erythema and induration at the Bacillus Calmette-Guerin and purified protein derivative inoculation sites. Pediatr Infect Dis J. 1987;6(6):576-8.

13. Uehara R, Yashiro M, Nakamura Y, Yanagawa H. Kawasaki disease in parents and children. Acta Paediatr. 2003;92(6):694-7.

14. Mojtahedzadeh s, Saket S, Shiari R, Shirvani F, Karimi A. [The relationship between history of ischemic heart disease in parents of children with Kawasaki Disease with severity of heart complications and disease recurrence in these children]. Yafte. 2011;12(4):61-6.

15. Lang B. Recognizing Kawasaki disease. Paediatr Child Health. 2001;6(9):638-43.

16. Friter BS, Lucky AW. The perineal eruption of Kawasaki syndrome. Arch Dermatol.1988;124(12):1805-10.

17. Yoskovitch A, Tewfik TL, Duffy CM, Moroz B. Head and neck manifestations of Kawasaki disease. Int J Pediatr Otorhinolaryngol. 2000;52(2):123-9.

18. Berard R, Scuccimarri R, Chedeville G. Leukonychia striata in Kawasaki disease. J Pediatr. 2008;152(6):889.

19. Kuniyuki S, Asada M. An ulcerated lesion at the BCG vaccination site during the course of Kawasaki disease. J Am Acad Dermatol. 1997;37(2 Pt 2):303-4.

20. Shiari R. Neurologic Manifestations of Childhood Rheumatic Diseases. Iran J Child Neurol. 2012;6(4):1-7.

21. Mason WH, Takahashi M. Kawasaki syndrome. Clin Infect Dis 1999;28(2):169-85.

22. Halimiasl A, Hosseini AH, Shiari R, Ghadamli P, Mojtahedzadeh S. . Concomitant Coronary Artery Aneurysm and Myocarditis as a Rare Manifestation of Kawasaki Disease: A Case Report. J Compr Ped. 2012;3(1):34-6.

23. Rowe PC, Quinlan A, Luke BK. Value of degenerative change in neutrophils as a diagnostic test for Kawasaki syndrome. J Pediatr. 1991;119(3):370-4.

24. Saket S, Mojtahedzadeh S, Karimi A, Shiari R, Shirvani F. [Relationship between electrolyte abnormalities, ESR, CRP and platelet count with severity of Kawasaki disease]. Yafte. 2009;11(3):5-14.

25. Latino GA, Manlhiot C, Yeung RS, Chahal N, McCrindle BW. Macrophage activation syndrome in the acute phase of Kawasaki disease. J Pediatr Hematol Oncol. 2010;32(7):527-31.

26. Mansour Ghnaee R, Shiari R, Karimi A, Armin S, Fa-himzad A Shiva F, et al. A case series report of Iranian children; Hemophagocytic Lymphohistiocytosis syndrome. Arch Pediatr Infect. 2012;1(1):31-5.
27. Sato YZ, Molkara DP, Daniels LB, Tremoulet AH, Shimizu C, Kanegaye JT, et al. Cardiovascular biomarkers in acute Kawasaki disease. Int J Cardiol. 2013;164(1):58-63.

28. Dahdah N, Siles A, Fournier A, Cousineau J, Delvin E, Saint-Cyr C, et al. Natriuretic peptide as an adjunctive diagnostic test in the acute phase of Kawasaki disease. Pediatr Cardiol. 2009;30(6):810-7.

29. Kato H. Cardiovascular involvement in Kawasaki disease: evaluation and natural history. Prog Clin Biol Res. 1987;250:277-86.

30. Fujiwara H, Hamashima Y. Pathology of the heart in Kawasaki disease. Pediatrics. 1978;61(1):100-7.

31. Dajani AS, Taubert KA, Gerber MA, Shulman ST, Ferrieri P, Freed $\mathrm{M}$, et al. Diagnosis and therapy of Kawasaki disease in children. Circulation. 1993;87(5):1776-80.

32. Capannari TE, Daniels SR, Meyer RA, Schwartz DC, Kaplan S. Sensitivity, specificity and predictive value of two-dimensional echocardiography in detecting coronary artery aneurysms in patients with Kawasaki disease. J Am Coll Cardiol. 1986;7(2):355-60.

33. Foster BJ, Bernard C, Drummond KN. Kawasaki disease complicated by renal artery stenosis. Arch Dis Child. 2000;83(3):253-5.

34. Newburger JW. Kawasaki disease: who is at risk? J Pediatr. 2000;137(2):149-52

35. Sonobe T, Kiyosawa N, Tsuchiya K, Aso S, Imada Y, Imai Y, et al. Prevalence of coronary artery abnormality in incomplete Kawasaki disease. Pediatr Int. 2007;49(4):421-6.

36. Chang FY, Hwang B, Chen SJ, Lee PC, Meng CC, Lu JH. Characteristics of Kawasaki disease in infants younger than 6 months of age. Pediatr Infect Dis J. 2006;25(3):241-4.

37. Gordon DJ, Sloan SR, de Jong JL. A pediatric case series of acute hemolysis after administration of intravenous immunoglobulin. Am J Hematol . 2009;84(771-2).

38. Lang B, Duffy CM. Controversies in the management of Kawasaki disease. Best Pract Res Clin Rheumatol. 2002;16(3):427-42.

39. Muta H, Ishii M, Egami K, Furui J, Sugahara Y, Akagi T, et al. Early intravenous gamma-globulin treatment for Kawasaki disease: the nationwide surveys in Japan. J Pediatr. 2004;144(4):496-9.

40. Freeman AF, Shulman ST. Refractory Kawasaki disease. Pediatr Infect Dis J . 2004;23(5):463-4.

41. Wooditch AC, Aronoff SC. Effect of initial corticosteroid therapy on coronary artery aneurysm formation in Kawasaki disease: a meta-analysis of 862 children. Pediatrics. 2005;116(4):989-95.

42. Mori M, Imagawa T, Katakura S, Miyamae T, Okuyama K, Ito S, et al. Efficacy of plasma exchange therapy for Kawasaki disease intractable to intravenous gamma-globulin. Mod Rheumatol. 2004;14(1):43-7.

43. McCandless RT, Minich LL, Tani LY, Williams RV. Does abciximab promote coronary artery remodeling in patients with Kawasaki disease? Am J Cardiol. 2010;105(11):1625-8.

44. Burns JC, Best BM, Mejias A, Mahony L, Fixler DE, Jafri HS, et al. Infliximab treatment of intravenous immunoglobulin-resistant Kawasaki disease. J Pediatr. 2008;153(6):833-8.

45. Burns JC, Mason WH, Hauger SB, Janai H, Bastian JF, Wohrley JD, et al. Infliximab treatment for refractory Kawasaki syndrome. $J$ Pediatr. 2005;146(5):662-7.

46. Raman V, Kim J, Sharkey A, Chatila T. Response of refractory Kawasaki disease to pulse steroid and cyclosporine A therapy. Pediatr Infect Dis J. 2001;20(6):635-7.

47. Ahn SY, Kim DS. Treatment of intravenous immunoglobulin-resistant Kawasaki disease with methotrexate. Scand J Rheumatol. 2005;34(2):136-9.

48. Wallace CA, French JW, Kahn SJ. Initial intravenous gammaglobulin treatment failure in Kawasaki disease. Pediatrics. 2000;105. 